Cambridge University Press

978-1-107-12392-2 - Magnetohydrodynamics of Laboratory and Astrophysical Plasmas

Hans Goedbloed, Rony Keppens, Stefaan Poedts

Frontmatter

More Information

\title{
MAGNETOHYDRODYNAMICS \\ of Laboratory and Astrophysical Plasmas
}

\begin{abstract}
With $90 \%$ of visible matter in the Universe existing in the plasma state, an understanding of magnetohydrodynamics is essential for anyone looking to understand solar and astrophysical processes, from stars to accretion discs and galaxies; as well as laboratory applications focused on harnessing controlled fusion energy.

This introduction to magnetohydrodynamics brings together the theory of plasma behavior with advanced topics including the applications of plasma physics to thermonuclear fusion and plasmaastrophysics. Topics covered include streaming and toroidal plasmas, nonlinear dynamics, modern computational techniques, incompressible plasma turbulence and extreme transonic and relativistic plasma flows. The numerical techniques needed to apply magnetohydrodynamics are explained, allowing the reader to move from theory to application and exploit the latest algorithmic advances. Bringing together two previous volumes: Principles of Magnetohydrodynamics and Advanced Magnetohydrodynamics, and completely updated with new examples, insights and applications, this volume constitutes a comprehensive reference for students and researchers interested in plasma physics, astrophysics and thermonuclear fusion.
\end{abstract}

HANS GOEDB LOED is Advisor of the Dutch Institute for Fundamental Energy Research (DIFFER), and Professor Emeritus of Theoretical Plasma Physics at Utrecht University. He has been a visiting scientist at laboratories in the Soviet Union, the United States, Brazil and Europe. He has taught at Campinas, Rio de Janeiro, São Paulo, Massachusetts Institute of Technology, Katholieke Universiteit Leuven, Amsterdam Free University and Utrecht University. For many years he coordinated an interdisciplinary and large-scale computational effort with the Dutch Science Organisation on 'Fast Changes in Complex Flows.'

RONY KEPPENS is Professor and Division Chair at the Centre for mathematical PlasmaAstrophysics, Katholieke Universiteit Leuven. He headed numerical plasma dynamics teams at FOM-Institute for Plasma Physics 'Rijnhuizen' (now DIFFER) and Leuven and frequently lectures on computational methods in astrophysics. His career started at the National Center for Atmospheric Research, Boulder, and the Kiepenheuer Institute for Solar Physics, Freiburg. He held a professorship at Utrecht University and a concurrent professorship at Nanjing University. His expertise ranges from solar to high-energy astrophysics and includes parallel computing and grid-adaptivity.

Stefan poedts is Professor and Chair of the Department of Mathematics at Katholieke Universiteit Leuven. He was a post-doctoral researcher at the Max Planck Institute for Plasma Physics, Garching, a senior researcher at the FOM-Institute for plasma physics 'Rijnhuizen', and research associate at the Centre for mathematical Plasma-Astrophysics, Katholieke Universiteit Leuven. His research interests include solar physics, space weather, thermonuclear fusion, MHD (in)stability, and multi-fluid modelling. He teaches basic math courses, and advanced courses on plasma physics of the Sun and numerical simulation. 
Cambridge University Press

978-1-107-12392-2 - Magnetohydrodynamics of Laboratory and Astrophysical Plasmas

Hans Goedbloed, Rony Keppens , Stefaan Poedts

Frontmatter

More Information 


\title{
MAGNETOHYDRODYNAMICS
}

of Laboratory and Astrophysical Plasmas

\author{
HANS GOEDBLOED
}

Dutch Institute for Fundamental Energy Research (DIFFER)

\section{RONY KEPPENS}

Centre for mathematical Plasma-Astrophysics,

Katholieke Universiteit Leuven

\section{STEFAAN POEDTS}

Centre for mathematical Plasma-Astrophysics, Katholieke Universiteit Leuven 
Cambridge University Press

978-1-107-12392-2 - Magnetohydrodynamics of Laboratory and Astrophysical Plasmas

Hans Goedbloed, Rony Keppens, Stefaan Poedts

Frontmatter

More Information

\section{CAMBRIDGE UNIVERSITY PRESS}

University Printing House, Cambridge CB2 8BS, United Kingdom

One Liberty Plaza, 20th Floor, New York, NY 10006, USA

477 Williamstown Road, Port Melbourne, VIC 3207, Australia

314-321, 3rd Floor, Plot 3, Splendor Forum, Jasola District Centre, New Delhi - 110025, India

79 Anson Road, \#06-04/06, Singapore 079906

Cambridge University Press is part of the University of Cambridge.

It furthers the University's mission by disseminating knowledge in the pursuit of education, learning, and research at the highest international levels of excellence.

www.cambridge.org

Information on this title: www.cambridge.org/9781107123922

DOI: $10.1017 / 9781316403679$

(C) Hans Goedbloed, Rony Keppens, and Stefaan Poedts 2019

This publication is in copyright. Subject to statutory exception and to the provisions of relevant collective licensing agreements, no reproduction of any part may take place without the written permission of Cambridge University Press.

First published 2019

Printed in the United Kingdom by TJ International Ltd. Padstow Cornwall

A catalogue record for this publication is available from the British Library.

Library of Congress Cataloging-in-Publication Data

Names: Goedbloed, J. P., 1940- author. | Keppens, Rony, author. | Poedts, Stefaan, 1962- author.

Title: Magnetohydrodynamics of laboratory and astrophysical plasmas / J.P.

Goedbloed (Dutch Institute for Fundamental Energy Research (DIFFER)), Rony

Keppens (Katholieke Universiteit Leuven, Belgium), Stefaan Poedts

(Katholieke Universiteit Leuven, Belgium).

Description: Cambridge ; New York, NY : Cambridge University Press, 2019.

Includes bibliographical references and index.

Identifiers: LCCN 2018029282 | ISBN 9781107123922

Subjects: LCSH: Magnetohydrodynamics. | Plasma astrophysics. | Astrophysics.

Classification: LCC QC718.5.M36 G63945 2019 |DDC 538/.6-dc23

LC record available at https://lccn.loc.gov/2018029282

ISBN 978-1-107-12392-2 Hardback

Cambridge University Press has no responsibility for the persistence or accuracy of URLs for external or third-party internet websites referred to in this publication and does not guarantee that any content on such websites is, or will remain, accurate or appropriate. 
To Antonia, 陆蓉 (Rong Lu) and Małgosia 
Cambridge University Press

978-1-107-12392-2 - Magnetohydrodynamics of Laboratory and Astrophysical Plasmas

Hans Goedbloed, Rony Keppens , Stefaan Poedts

Frontmatter

More Information 
Cambridge University Press

978-1-107-12392-2 - Magnetohydrodynamics of Laboratory and Astrophysical Plasmas

Hans Goedbloed, Rony Keppens, Stefaan Poedts

Frontmatter

More Information

\section{Contents}

Preface

page xvii

Part I Plasma Physics Preliminaries 1

1 Introduction 3

1.1 Motivation 3

1.2 Thermonuclear fusion and plasma confinement 4

1.2.1 Fusion reactions 4

1.2.2 Conditions for fusion 6

1.2.3 Magnetic confinement and tokamaks 9

1.3 Astrophysical plasmas $\quad 11$

$\begin{array}{lll}\text { 1.3.1 Celestial mechanics } & 11\end{array}$

$\begin{array}{lll}1.3 .2 & \text { Astrophysics } & 13\end{array}$

$\begin{array}{lll}1.3 .3 & \text { Plasmas enter the stage } & 15\end{array}$

$\begin{array}{lll}\text { 1.3.4 The standard view of nature } & 17\end{array}$

1.4 Definitions of the plasma state 19

$\begin{array}{lll}\text { 1.4.1 Microscopic definition of plasma } & 19\end{array}$

1.4.2 Macroscopic approach to plasma 23

$\begin{array}{lll}1.5 & \text { Literature and exercises } & 24\end{array}$

$2 \quad$ Elements of plasma physics $\quad 27$

2.1 Theoretical models $\quad 27$

2.2 Single particle motion $\quad 27$

2.2.1 Cyclotron motion 27

2.2.2 Excursion: Basic equations of electrodynamics and mechanics 30

2.2.3 Drifts, adiabatic invariants 33

$2.3 \quad$ Kinetic plasma theory 38

2.3.1 Boltzmann equation and moment reduction 38

2.3.2 Collective phenomena: plasma oscillations $\quad 43$

2.3.3 Landau damping 46

$2.4 \quad$ Fluid description $\quad 52$

2.4.1 From the two-fluid to the MHD description of plasmas 53

2.4.2 Alfvén waves 57

2.4.3 Equilibrium and stability 59 
Cambridge University Press

978-1-107-12392-2 - Magnetohydrodynamics of Laboratory and Astrophysical Plasmas

Hans Goedbloed, Rony Keppens , Stefaan Poedts

Frontmatter

More Information

2.5 In conclusion

$2.6 \quad$ Literature and exercises 64

3 'Derivation' of the macroscopic equations ${ }^{\star}$

3.1 Two approaches ${ }^{\star} \quad 66$

3.2 Kinetic equations ${ }^{\star} \quad 67$

3.2.1 Boltzmann equation ${ }^{\star} \quad 67$

3.2.2 Moments of the Boltzmann equation ${ }^{\star} \quad 70$

3.2.3 Thermal fluctuations and transport ${ }^{\star} \quad 72$

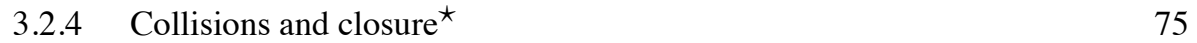

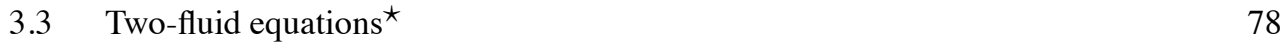

3.3.1 Electron-ion plasma* 78

3.3.2 The classical transport coefficients ${ }^{\star} \quad 79$

3.3.3 Dissipative versus ideal fluids ${ }^{\star} \quad 83$

3.3.4 Excursion: waves in two-fluid plasmas ${ }^{\star} \quad 86$

3.4 One-fluid equations ${ }^{\star} \quad 95$

3.4.1 Maximal ordering for MHD* 95

3.4.2 Resistive and ideal MHD equations ${ }^{\star} \quad 99$

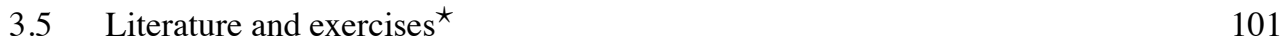

Part II Basic Magnetohydrodynamics 103

4 The MHD model $\quad 105$

4.1 The ideal MHD equations 105

$\begin{array}{ll}\text { 4.1.1 Postulating the basic equations } & 105\end{array}$

$\begin{array}{lll}4.1 .2 & \text { Scale independence } & 110\end{array}$

4.1.3 A crucial question $\quad 112$

4.2 Magnetic flux 113

$\begin{array}{lll}4.2 .1 & \text { Flux tubes } & 113\end{array}$

4.2.2 Global magnetic flux conservation $\quad 114$

4.3 Conservation laws 116

4.3.1 Conservation form of the MHD equations 116

$\begin{array}{ll}\text { 4.3.2 Global conservation laws } & 118\end{array}$

4.3.3 Local conservation of magnetic flux $\quad 121$

$\begin{array}{ll}\text { 4.3.4 Magnetic helicity } & 124\end{array}$

4.4 Dissipative magnetohydrodynamics $\quad 128$

$\begin{array}{lll}\text { 4.4.1 Resistive MHD } & 128\end{array}$

4.4.2 (Non-)conservation form of the dissipative equations ${ }^{\star} \quad 131$

$\begin{array}{lll}4.5 & \text { Discontinuities } & 133\end{array}$

4.5.1 Shocks and jump conditions 133

4.5.2 Boundary conditions for plasmas with an interface 136

4.6 Model problems $\quad 138$

4.6.1 Laboratory plasmas (models I-III) 138

4.6.2 Energy conservation for interface plasmas $\quad 141$

4.6.3 Astrophysical plasmas (models IV-VI) 143 
Cambridge University Press

978-1-107-12392-2 - Magnetohydrodynamics of Laboratory and Astrophysical Plasmas

Hans Goedbloed, Rony Keppens , Stefaan Poedts

Frontmatter

More Information

4.7 Literature and exercises 144

$5 \quad$ Waves and characteristics 147

5.1 Physics and accounting 147

5.1.1 Introduction 147

$\begin{array}{ll}\text { 5.1.2 Sound waves } & 147\end{array}$

5.2 MHD waves 150

5.2.1 Symmetric representation in primitive variables $\quad 150$

5.2.2 Entropy wave and magnetic field constraint 152

5.2.3 Reduction to velocity representation: three waves 155

5.2.4 Dispersion diagrams 157

5.3 Phase and group diagrams 159

5.3.1 Basic concepts 159

5.3.2 Application to the MHD waves 161

5.3.3 Asymptotic properties 165

5.3.4 Self-gravity and contraction in homogeneous media ${ }^{\star} \quad 166$

5.4 Characteristics ${ }^{\star} \quad 169$

5.4.1 The method of characteristics ${ }^{\star} \quad 169$

5.4.2 Classification of partial differential equations ${ }^{\star} \quad 171$

5.4.3 Characteristics in ideal MHD* 173

$\begin{array}{lll}5.5 & \text { Literature and exercises } & 179\end{array}$

6 Spectral theory $\quad 181$

6.1 Stability: intuitive approach 181

6.1.1 Two viewpoints 181

6.1.2 Linearization and Lagrangian reduction 183

6.2 Force operator formalism 186

6.2.1 Equation of motion 186

6.2.2 Hilbert space 190

6.2.3 Proof of self-adjointness of the force operator 191

6.3 Spectral alternatives ${ }^{\star} \quad 196$

6.3.1 Mathematical intermezzo ${ }^{\star} \quad 196$

6.3.2 Initial value problem in $\mathrm{MHD}^{\star} \quad 198$

6.4 Quadratic forms and variational principles 200

6.4.1 Expressions for the potential energy 200

6.4.2 Hamilton's principle 202

6.4.3 Rayleigh-Ritz spectral variational principle 203

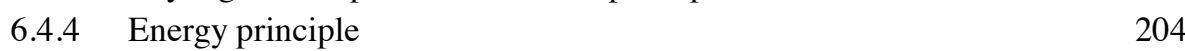

6.5 Further spectral issues 206

6.5.1 Normal modes and the energy principle ${ }^{\star} \quad 206$

$\begin{array}{ll}\text { 6.5.2 Proof of the energy principle } & 207\end{array}$

$\begin{array}{lll}6.5 .3 & \sigma \text {-stability } & 209\end{array}$

6.5.4 Returning to the two viewpoints 210

6.6 Extension to interface plasmas 213

$\begin{array}{ll}\text { 6.6.1 Boundary conditions at the interface } & 215\end{array}$ 
Cambridge University Press

978-1-107-12392-2 - Magnetohydrodynamics of Laboratory and Astrophysical Plasmas

Hans Goedbloed, Rony Keppens, Stefaan Poedts

Frontmatter

More Information

6.6.2 Self-adjointness for interface plasmas $\quad 218$

6.6.3 Extended variational principles $\quad 219$

6.6.4 Application to the Rayleigh-Taylor instability 221

$\begin{array}{lll}6.7 & \text { Literature and exercises } & 229\end{array}$

Part III Standard Model Applications 231

$7 \quad$ Waves and instabilities of inhomogeneous plasmas 233

$\begin{array}{lll}7.1 & \text { Hydrodynamics of the solar interior } & 233\end{array}$

7.1.1 Radiative equilibrium model 234

$\begin{array}{lll}\text { 7.1.2 Convection zone } & 237\end{array}$

7.2 Hydrodynamic waves and instabilities of a gravitating slab 239

7.2.1 Hydrodynamic wave equation 239

$\begin{array}{ll}\text { 7.2.2 Convective instabilities } & 241\end{array}$

$\begin{array}{lll}\text { 7.2.3 Gravito-acoustic waves } & 242\end{array}$

7.2.4 Helioseismology and MHD spectroscopy 245

7.3 MHD wave equation for a gravitating magnetized plasma slab 248

$\begin{array}{lll}\text { 7.3.1 Preliminaries } & 248\end{array}$

7.3.2 MHD wave equation for a gravitating slab 252

$\begin{array}{lll}\text { 7.3.3 Gravito-MHD waves } & 258\end{array}$

7.4 Continuous spectrum and spectral structure 265

$\begin{array}{lll}\text { 7.4.1 Singular differential equations } & 265\end{array}$

$\begin{array}{lll}\text { 7.4.2 Alfvén and slow continua } & 269\end{array}$

$\begin{array}{lll}\text { 7.4.3 Oscillation theorems } & 273\end{array}$

$\begin{array}{ll}\text { 7.4.4 Cluster spectra } & 278\end{array}$

7.5 Gravitational instabilities of a magnetized plasma slab 279

7.5.1 Energy principle for a gravitating plasma slab 280

7.5.2 Interchanges in shearless magnetic fields 283

7.5.3 Interchange instabilities in sheared magnetic fields 285

$\begin{array}{lll}7.6 & \text { Literature and exercises } & 289\end{array}$

8 Magnetic structures and dynamics of the solar system 292

8.1 Plasma dynamics in laboratory and nature 292

8.2 Solar magnetism 293

$\begin{array}{lll}\text { 8.2.1 The solar cycle } & 294\end{array}$

8.2.2 Magnetic structures in the solar atmosphere 300

$\begin{array}{ll}\text { 8.2.3 Inspiration from solar magnetism } & 309\end{array}$

8.2.4 Solar wind and heliosphere $\quad 309$

$\begin{array}{lll}8.3 & \text { Space weather } & 313\end{array}$

$\begin{array}{llr}\text { 8.3.1 Technological and economic implications } & 313\end{array}$

8.3.2 Coronal mass ejections $\quad 314$

8.3.3 Numerical modelling of space weather 317

8.3.4 Solar wind and planetary magnetospheres $\quad 320$

8.4 Perspective 321

$\begin{array}{lll}8.5 & \text { Literature and exercises } & 322\end{array}$ 
Cambridge University Press

978-1-107-12392-2 - Magnetohydrodynamics of Laboratory and Astrophysical Plasmas

Hans Goedbloed, Rony Keppens, Stefaan Poedts

Frontmatter

More Information

9 Cylindrical plasmas

9.1 Equilibrium of cylindrical plasmas

9.1.1 Diffuse plasmas $\quad 325$

9.1.2 Interface plasmas $\quad 329$

9.2 MHD wave equation for cylindrical plasmas 330

9.2.1 Derivation of the MHD wave equation for a cylinder 330

9.2.2 Boundary conditions for cylindrical interfaces 336

9.3 Spectral structure $\quad 339$

9.3.1 One-dimensional inhomogeneity 339

9.3.2 Cylindrical model problems $\quad 341$

$\begin{array}{ll}\text { 9.3.3 Cluster spectra } & 347\end{array}$

9.4 Stability of cylindrical plasmas $\quad 348$

9.4.1 Oscillation theorems for stability 348

9.4.2 Stability of plasmas with shearless magnetic fields 353

$\begin{array}{ll}\text { 9.4.3 Stability of force-free magnetic fields } & 357\end{array}$

9.4.4 Stability of the 'straight tokamak' 361

$\begin{array}{lll}9.5 & \text { Literature and exercises } & 369\end{array}$

10 Initial value problem and wave damping ${ }^{\star} \quad 372$

10.1 Implications of the continuous spectrum ${ }^{\star} \quad 372$

$\begin{array}{ll}10.2 & \text { Initial value problem } \\ & \end{array}$

10.2.1 Reduction to a one-dimensional representation ${ }^{\star} \quad 373$

10.2.2 Restoring the three-dimensional picture ${ }^{\star} \quad 376$

10.3 Damping of Alfvén waves ${ }^{\star} \quad 380$

$\begin{array}{ll}\text { 10.3.1 Green's function } & 381\end{array}$

$\begin{array}{ll}10.3 .2 \text { Spectral cuts } & 384\end{array}$

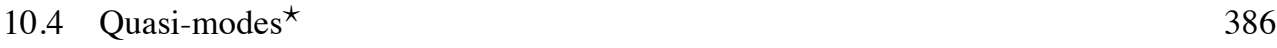

$\begin{array}{lll}10.5 & \text { Leaky modes } & 392\end{array}$

10.6 Literature and exercises ${ }^{\star} \quad 397$

11 Resonant absorption and wave heating $\quad 399$

11.1 Ideal MHD theory of resonant absorption 399

11.1.1 Analytical solution of a simple model problem 399

11.1.2 Role of the singularity 405

11.1.3 Resonant 'absorption' versus resonant 'dissipation' 414

11.2 Heating and wave damping in tokamaks and coronal loops 417

$\begin{array}{lll}\text { 11.2.1 Tokamaks } & 417\end{array}$

11.2.2 Coronal loops and arcades $\quad 418$

11.2.3 Numerical analysis of resonant absorption $\quad 419$

11.3 Alternative excitation mechanisms 423

11.3.1 Foot point driving $\quad 424$

$\begin{array}{ll}\text { 11.3.2 Phase mixing } & 427\end{array}$

11.3.3 Applications to solar and magnetospheric plasmas $\quad 428$

11.4 Literature and exercises $\quad 432$ 
Cambridge University Press

978-1-107-12392-2 - Magnetohydrodynamics of Laboratory and Astrophysical Plasmas

Hans Goedbloed, Rony Keppens, Stefaan Poedts

Frontmatter

More Information

Part IV Flow and Dissipation

12 Waves and instabilities of stationary plasmas

12.1 Laboratory and astrophysical plasmas

12.1.1 Grand vision: magnetized plasma on all scales!

12.1.2 Laboratory and astrophysical plasmas

440

12.1.3 Interchanges and the Parker instability 441

12.2 Spectral theory of stationary plasmas 445

12.2.1 Plasmas with background flow 445

12.2.2 Frieman-Rotenberg formulation 448

12.2.3 Self-adjointness of the generalized force operator ${ }^{\star} \quad 453$

12.2.4 Energy conservation and stability 456

12.3 The Spectral Web $\quad 462$

12.3.1 Opening up the boundaries 462

12.3.2 Oscillation theorems in the complex plane 466

$\begin{array}{lll}12.4 & \text { Literature and exercises } & 471\end{array}$

13 Shear flow and rotation 473

13.1 Spectral theory of plane plasmas with shear flow 473

13.1.1 Gravito-MHD wave equation for plane plasma flow 473

13.1.2 Kelvin-Helmholtz instabilities in interface plasmas 478

13.1.3 Continua and the real oscillation theorem 480

13.1.4 Spectral Web and the complex oscillation theorem 484

13.2 Analysis of flow-driven instabilities in plane plasmas 486

13.2.1 Rayleigh-Taylor instabilities of magnetized plasmas 488

13.2.2 Kelvin-Helmholtz instabilities of ordinary fluids 489

13.2.3 Combined instabilities of magnetized plasmas 494

13.3 Spectral theory of rotating plasmas 498

13.3.1 MHD wave equation for cylindrical flow in 3D 498

13.3.2 Reduction to a second order differential equation 500

$\begin{array}{ll}\text { 13.3.3 Singular expansions } & 502\end{array}$

13.3.4 Doppler-Coriolis shift and solution path 505

13.4 Rayleigh-Taylor instabilities in rotating theta-pinches 506

13.4.1 Hydrodynamic modes $(k=0) \quad 507$

13.4.2 Magnetohydrodynamic modifications $(k \neq 0) \quad 511$

13.5 Magneto-rotational instability in accretion discs 513

$\begin{array}{lll}\text { 13.5.1 Analytical preliminaries } & 514\end{array}$

13.5.2 Numerical Spectral Web solutions $\quad 518$

13.6 Literature and exercises $\quad 523$

14 Resistive plasma dynamics $\quad 525$

14.1 Plasmas with dissipation $\quad 525$

14.1.1 Conservative versus dissipative dynamical systems $\quad 525$

14.1.2 Stability of force-free magnetic fields: a trap $\quad 525$

14.2 Resistive instabilities $\quad 532$

$\begin{array}{lll}\text { 14.2.1 Basic equations } & 532\end{array}$ 
Cambridge University Press

978-1-107-12392-2 - Magnetohydrodynamics of Laboratory and Astrophysical Plasmas

Hans Goedbloed, Rony Keppens, Stefaan Poedts

Frontmatter

More Information

Contents

14.2.2 Tearing modes $\quad 534$

14.2.3 Resistive interchange modes $\quad 543$

14.3 Resistive spectrum 544

14.3.1 Resistive wall mode $\quad 544$

14.3.2 Spectrum of homogeneous plasma $\quad 548$

14.3.3 Spectrum of inhomogeneous plasma $\quad 551$

14.4 Reconnection $\quad 554$

14.4.1 Reconnection in a 2D Harris sheet $\quad 554$

14.4.2 Petschek reconnection 558

14.4.3 Kelvin-Helmholtz induced tearing instabilities $\quad 559$

14.4.4 Extended MHD and reconnection $\quad 560$

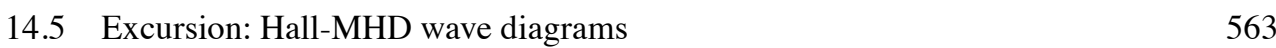

14.6 Literature and exercises $\quad 566$

15 Computational linear MHD $\quad 569$

15.1 Spatial discretization techniques $\quad 569$

$\begin{array}{lll}\text { 15.1.1 } & \text { Basic concepts for discrete representations } & 571\end{array}$

$\begin{array}{lll}\text { 15.1.2 } & \text { Finite difference methods } & 572\end{array}$

15.1.3 Finite element method 576

15.1.4 Spectral methods $\quad 583$

15.1.5 Mixed representations $\quad 586$

15.2 Linear MHD: boundary value problems 588

15.2.1 Linearized MHD equations $\quad 589$

15.2.2 Steady solutions to linearly driven problems $\quad 590$

15.2.3 MHD eigenvalue problems $\quad 593$

15.2.4 Extended MHD examples $\quad 594$

15.3 Linear MHD: initial value problems 599

15.3.1 Temporal discretizations: explicit methods $\quad 599$

15.3.2 Disparateness of MHD time scales $\quad 606$

15.3.3 Temporal discretizations: implicit methods 606

15.3.4 Applications: linear MHD evolutions $\quad 608$

$\begin{array}{ll}15.4 & \text { Concluding remarks }\end{array} 612$

$\begin{array}{lll}15.5 & \text { Literature and exercises } & 612\end{array}$

$\begin{array}{lll}\text { Part V Toroidal Geometry } & 615\end{array}$

16 Static equilibrium of toroidal plasmas $\quad 617$

$\begin{array}{lll}16.1 & \text { Axi-symmetric equilibrium } & 617\end{array}$

$\begin{array}{ll}\text { 16.1.1 Equilibrium in tokamaks } & 617\end{array}$

$\begin{array}{ll}\text { 16.1.2 Magnetic field geometry } & 621\end{array}$

16.1.3 Cylindrical limits $\quad 624$

16.1.4 Global confinement and parameters $\quad 627$

16.2 Grad-Shafranov equation 635

16.2.1 Derivation of the Grad-Shafranov equation 635

16.2.2 Large aspect ratio expansion: internal solution 637 
Cambridge University Press

978-1-107-12392-2 - Magnetohydrodynamics of Laboratory and Astrophysical Plasmas

Hans Goedbloed, Rony Keppens, Stefaan Poedts

Frontmatter

More Information

16.2.3 Large aspect ratio expansion: external solution

16.3 Exact equilibrium solutions

16.3.1 Poloidal flux scaling

16.3.2 Soloviev equilibrium

16.3.3 Numerical equilibria ${ }^{\star}$

16.4 Extensions

16.4.1 Toroidal rotation

16.4.2 Gravitating plasma equilibria ${ }^{\star}$

662

16.4.3 Challenges

16.5 Literature and exercises

17 Linear dynamics of static toroidal plasmas

17.1 "Ad more geometrico"

17.1.1 Alfvén wave dynamics in toroidal geometry

17.1.2 Coordinates and mapping

17.1.3 Geometrical-physical characteristics

17.2 Analysis of waves and instabilities in toroidal geometry

17.2.1 Spectral wave equation

17.2.2 Spectral variational principle

17.2.3 Alfvén and slow continuum modes

677

17.2.4 Poloidal mode coupling

680

17.2.5 Alfvén and slow ballooning modes

17.3 Computation of waves and instabilities in tokamaks

17.3.1 Ideal MHD versus resistive MHD in computations

690

17.3.2 Internal modes

695

17.3.3 Edge localized modes

697

17.3.4 Toroidal Alfvén eigenmodes and MHD spectroscopy 701

17.4 Literature and exercises

18.2 Axi-symmetric equilibrium of transonic stationary states ${ }^{\star}$

18.2.2 Equilibrium variational principle and rescaling ${ }^{\star} \quad 712$

18.2.3 Elliptic and hyperbolic flow regimes ${ }^{\star} \quad 715$

18.2.4 Expansion of the equilibrium in small toroidicity ${ }^{\star} \quad 716$

$\begin{array}{ll}18.3 \text { Equations for the continuous spectrum }{ }^{\star} & 722\end{array}$

$\begin{array}{ll}\text { 18.3.1 Reduction for straight-field-line coordinates } & 722\end{array}$

18.3.2 Continua of poloidally and toroidally rotating plasmas ${ }^{\star} \quad 725$

18.3.3 Analysis of trans-slow continua for small toroidicity ${ }^{\star} \quad 731$

18.4 Trans-slow continua in tokamaks and accretion discs ${ }^{\star} \quad 737$

18.4.1 Tokamaks and magnetically dominated accretion discs ${ }^{\star} \quad 738$

$\begin{array}{ll}\text { 18.4.2 Gravity dominated accretion discs } & 740\end{array}$

18.4.3 Trans-slow Alfvén continuum instabilities 742

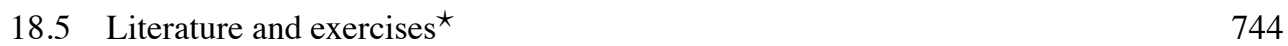


Cambridge University Press

978-1-107-12392-2 - Magnetohydrodynamics of Laboratory and Astrophysical Plasmas

Hans Goedbloed, Rony Keppens, Stefaan Poedts

Frontmatter

More Information

\section{Contents}

Part VI Nonlinear Dynamics

19 Turbulence in incompressible magneto-fluids

19.1 Incompressible hydrodynamics preliminaries $\quad 749$

19.1.1 The incompressible hydro model 749

19.1.2 Two-dimensional formulations $\quad 751$

19.1.3 'Wave' analysis for incompressible Euler 751

19.1.4 Energy equation and Kolmogorov scaling 753

19.1.5 Selected numerical examples 756

19.2 Incompressible magnetohydrodynamics 758

19.2.1 Governing equations $\quad 758$

19.2.2 Elsässer formulation $\quad 759$

19.2.3 Kinematic MHD modelling 760

19.2.4 Dynamo aspects $\quad 761$

19.3 Waves in incompressible MHD 764

19.3.1 Linear wave analysis $\quad 765$

19.3.2 Nonlinear wave solutions and conservation laws 766

19.3.3 MHD turbulence scaling laws $\quad 768$

19.4 Incompressible MHD simulations 771

19.4.1 Structure formation in incompressible MHD studies 772

$\begin{array}{ll}\text { 19.4.2 Dynamo aspects continued } & 774\end{array}$

19.5 Extension to compressible MHD and concluding remarks 776

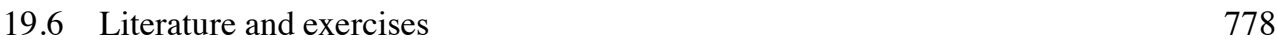

20 Computational nonlinear MHD $\quad 780$

20.1 General considerations for nonlinear conservation laws $\quad 780$

20.1.1 Conservative versus primitive variable formulations $\quad 780$

20.1.2 Scalar conservation law and the Riemann problem 786

20.1.3 Numerical discretizations for scalar conservation $\quad 790$

20.1.4 Finite volume treatments 796

20.2 Upwind-like finite volume treatments for one-dimensional MHD 797

20.2.1 The Godunov method 798

20.2.2 A robust shock-capturing method: TVDLF 802

20.2.3 Approximate Riemann solver schemes 807

20.2.4 Simulating 1D MHD Riemann problems $\quad 811$

20.3 Multi-dimensional MHD computations $\quad 813$

20.3.1 $\nabla \cdot \mathbf{B}=0$ condition for shock-capturing schemes $\quad 814$

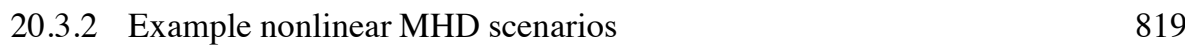

20.3.3 Alternative numerical methods $\quad 822$

20.4 Implicit approaches for extended MHD simulations $\quad 827$

20.4.1 Semi-implicit methods $\quad 828$

20.4.2 Simulating ideal and resistive instabilities $\quad 832$

20.4.3 Global simulations for tokamak plasmas 833

20.5 Literature and exercises $\quad 834$ 
Cambridge University Press

978-1-107-12392-2 - Magnetohydrodynamics of Laboratory and Astrophysical Plasmas

Hans Goedbloed, Rony Keppens, Stefaan Poedts

Frontmatter

More Information

21 Transonic MHD flows and shocks $\quad 837$

21.1 Transonic flows $\quad 837$

$\begin{array}{lll}21.1 .1 & \text { Characteristics and shocks } & 838\end{array}$

$\begin{array}{ll}21.1 .2 \text { Gas dynamic shocks } & 840\end{array}$

$\begin{array}{lll}21.1 .3 & \text { Misnomers } & 845\end{array}$

21.2 MHD shock conditions $\quad 846$

21.2.1 MHD discontinuities without mass flow 846

21.2.2 MHD discontinuities with mass flow 848

$\begin{array}{ll}\text { 21.2.3 Slow, intermediate and fast shocks } & 852\end{array}$

21.3 Advanced classification of MHD shocks $\quad 854$

21.3.1 Distilled shock conditions $\quad 854$

21.3.2 Time reversal duality $\quad 859$

21.3.3 Angular dependence of MHD shocks * 865

$\begin{array}{ll}\text { 21.3.4 Observational considerations of MHD shocks } & 870\end{array}$

$\begin{array}{lll}21.4 & \text { Example astrophysical transonic flows } & 871\end{array}$

21.5 Literature and exercises $\quad 876$

22 Ideal MHD in special relativity

22.1 Four-dimensional space-time: special relativistic concepts $\quad 879$

22.1.1 Space-time coordinates and Lorentz transformations $\quad 880$

22.1.2 Four-vectors in flat space-time and invariants $\quad 882$

22.1.3 Relativistic gas dynamics and stress-energy tensor $\quad 885$

22.1.4 Sound waves and shock relations in relativistic gases $\quad 889$

22.2 Electromagnetism and special relativistic MHD 895

22.2.1 Electromagnetic field tensor and Maxwell's equations 895

22.2.2 Ideal MHD in special relativity 900

22.2.3 Wave dynamics in a homogeneous plasma 902

22.2.4 Shock conditions in relativistic MHD 906

22.3 Computing relativistic magnetized plasma dynamics 908

22.3.1 Numerical challenges from relativistic MHD 910

22.3.2 Pulsar Wind Nebulae modelling $\quad 911$

22.4 Outlook: General relativistic MHD simulations 915

22.5 Literature and exercises $\quad 916$

Appendices $\quad 919$

A Vectors and coordinates $\quad 919$

A.1 Vector identities $\quad 919$

A.2 Vector expressions in orthogonal coordinates 920

A.3 Vector expressions in non-orthogonal coordinates 927

B Tables of physical quantities $\quad 931$

References $\quad 937$

$\begin{array}{ll}\text { Index } & 964\end{array}$ 
Cambridge University Press

978-1-107-12392-2 - Magnetohydrodynamics of Laboratory and Astrophysical Plasmas

Hans Goedbloed, Rony Keppens , Stefaan Poedts

Frontmatter

More Information

\section{Preface}

This book describes the two main applications of plasma physics, laboratory research on thermonuclear fusion energy and plasma-astrophysics of astronomical systems, from the single viewpoint of magnetohydrodynamics (MHD). This provides effective methods and insights for the interpretation of plasma phenomena on virtually all scales, ranging from the laboratory to the Universe. The key issue is understanding the complexities of plasma dynamics in extended magnetic structures. In the first half of the book, based on a revision of the previous volume Principles of Magnetohydrodynamics [1], the classical MHD model is developed in great detail without omitting steps in the derivations. This necessitated restriction to ideal dissipationless plasmas, in static equilibrium and inhomogeneous in one direction. In the second half of the book, based on a revision of the previous Advanced Magnetohydrodynamics [2], these restrictions are relaxed one by one: introducing stationary background flows, dissipation, two-dimensional toroidal geometry, linear and nonlinear computational techniques, turbulence, transonic flows and relativity. These topics transform the subject into a vital new area with numerous applications in laboratory, space and astrophysical plasmas. It is impossible to treat all topics that actually belong to the field of advanced MHD. Fortunately, books or chapters of books exist on some of those topics, like dynamos [444, 475, 174, 533], solar magnetohydrodynamics [510], chaos [649], stellarators [185], spheromaks [48] and anomalous transport $[33,662]$.

Inevitably, with the distinction between topics for Chapters 1-11 (mostly ideal linear phenomena described by self-adjoint linear operators) and Chapters 12-22 (mostly non-ideal and nonlinear phenomena), the difference between 'basic' and 'advanced' levels of magnetohydrodynamics could not strictly be maintained. The logical order required a quite advanced derivation of the MHD equations from kinetic theory (Chapter 3) at an early stage, different sections on advanced topics interspersed throughout the book, and rather complicated analyses of the initial value problem (Chapter 10) and flow in toroidal systems (Chapter 18). These parts are marked by a star $\left(^{\star}\right)$ and can be skipped on a first study of the book. The same applies to text put in small print, in between triangles $(\triangleright \cdots \triangleleft)$, usually containing tedious derivations or advanced material. The serious student is advised though not to skip the Exercises, which are also put in small print for typographical reasons. Frequent use of the vector expressions and tables of the Appendices is encouraged. Magnetohydrodynamics can only be mastered through intense practice. 
Cambridge University Press

978-1-107-12392-2 - Magnetohydrodynamics of Laboratory and Astrophysical Plasmas

Hans Goedbloed, Rony Keppens, Stefaan Poedts

Frontmatter

More Information

An overview of the subject matter of the different parts and chapters of this book may help the reader to find his way.

\section{Part I (Plasma physics preliminaries)}

- Chapter 1 gives an introduction to laboratory fusion and astrophysical plasmas, and formulates provisional microscopic and macroscopic definitions of the plasma state.

- Chapter 2 discusses the three complementary points of view of single particle motion, kinetic theory and fluid description. The corresponding theoretical models provide the opportunity to introduce some of the basic concepts of plasma physics.

- Chapter 3 gives the 'derivation' of the macroscopic equations from the kinetic equations. Quotation marks because a fully satisfactory derivation can not be given at present in view of the largely unknown contribution of turbulent transport processes. The presentation provides some impression of the limitations of the macroscopic model.

\section{Part II (Basic magnetohydrodynamics)}

- Chapter 4 defines the MHD model and introduces the concept of scale independence. The central importance of the conservation laws is discussed at length. Based on this, the similarities and differences of laboratory and astrophysical plasmas are articulated in terms of a set of generic boundary value problems.

- Chapter 5 derives the basic MHD waves and describes their properties, with an eye on their role in spectral analysis and computational MHD. The theory of characteristics is introduced as a way to describe the propagation of nonlinear disturbances.

- Chapter 6 treats the subject of waves and instabilities from the unifying point of view of spectral theory. The force operator formulation and the energy principle are extensively discussed. The analogy with quantum mechanics is pointed out and exploited.

\section{Part III (Standard model applications)}

- Chapter 7 applies the spectral analysis of Chapter 6 to inhomogeneous plasmas in a plane slab. The wave equation for gravito-MHD waves is derived and solved in various limits. Here, all the intricacies of the subject enter: continuous spectra, damping of Alfvén waves, local instabilities, etc. The topic of MHD spectroscopy is shown to hold great promise for the diagnostics of plasma dynamics.

- Chapter 8 introduces the enormous variety of magnetic phenomena in astrophysics, in particular for the solar system (dynamo, solar wind, space weather, etc.), and provides basic examples of plasma dynamics worked out in later chapters.

- Chapter 9 is the cylindrical counterpart of Chapter 7, with a wave equation describing the various waves and instabilities. It presents the stability analysis of diffuse cylindrical plasmas (classical pinches and present tokamaks) from the spectral perspective.

- Chapter 10 solves the initial value problem for one-dimensional inhomogeneous MHD and the associated damping due to the continuous spectrum.

- Chapter 11 discusses resonant absorption and phase mixing in the context of heating mechanisms of laboratory plasmas, and solar or stellar coronae. Sunspot seismology is introduced as another example of MHD spectroscopy. 
Cambridge University Press

978-1-107-12392-2 - Magnetohydrodynamics of Laboratory and Astrophysical Plasmas

Hans Goedbloed, Rony Keppens, Stefaan Poedts

Frontmatter

More Information

\section{Part IV (Flow and dissipation)}

- Chapter 12 initiates the most urgent extension of the theory of Chapters 1-10, viz. waves and instabilities in plasmas with stationary background flows, a theme of great interest for laboratory fusion and astrophysical plasma research. The old problem of how to find the complex eigenvalues of stationary plasmas is solved by means of the new method of constructing the Spectral Web in the complex plane.

- Chapter 13 applies the new theory of the Spectral Web to the two classical topics of shear flow in plane plasma slabs, including the Kelvin-Helmholtz instability, and rotation in cylindrical plasmas, including the magneto-rotational instability.

- Chapter 14 treats the considerable modification of plasma dynamics when resistivity is introduced in the MHD description, both in the linear domain of spectral theory and in the nonlinear domain of reconnection.

- Chapter 15 introduces the basic techniques of computational MHD, the discretization techniques, the methods of time stepping, etc. It thus provides the modern techniques needed to solve for the dynamics of plasmas in complicated magnetic geometries.

\section{Part V (Toroidal geometry)}

- Chapter 16 presents the classical theory of static equilibrium of toroidal plasmas, a topic of central interest in fusion research of tokamaks.

- Chapter 17 concerns the spectral theory of waves and instabilities in toroidal equilibria, again a central topic in tokamak research. Because of this important application, this part of MHD spectral theory is the most developed one, also with respect to comparison with experimental data. This activity is called MHD spectroscopy.

- Chapter 18 introduces the theory of transonic equilibria and spectral theory of toroidal equilibria rotating in both directions, a subject of great interest but still in its infancy.

Part VI (Nonlinear dynamics)

- Chapter 19 introduces the topic of 2D turbulence in magneto-fluids by deriving the scaling laws for MHD turbulence and presenting the high performance computing efforts needed to resolve the structures that occur.

- Chapter 20 presents the counterpart of Chapter 15 by introducing the numerical methods for nonlinear MHD, in particular for plasmas with large background flows, applied in the last two chapters of this book.

- Chapter 21 discusses the MHD shock conditions from a new perspective, scale independence leading to time reversal duality, and it introduces some of the important areas of application of nonlinear MHD, viz. astrophysical winds and transonic flows.

- Chapter 22 introduces special relativistic MHD, in particular the linear waves and nonlinear shocks that occur at relativistic speeds. The books ends with applications to astrophysical phenomena, like relativistic jets, and thus completes the panorama of the tremendously exciting field of magnetohydrodynamics dominated by flows.

The Appendices provide the essentials of the two indispensable tools for MHD calculations, vector relations and orders of magnitude of the plasma parameters. 
Cambridge University Press

978-1-107-12392-2 - Magnetohydrodynamics of Laboratory and Astrophysical Plasmas

Hans Goedbloed, Rony Keppens, Stefaan Poedts

Frontmatter

More Information

We wish to acknowledge Guido Huysmans, Jelle Kaastra, Giovanni Lapenta, Sasha Lifschitz, Zakaria Meliani, Gábor Tóth, Ronald Van der Linden, Oliver Porth, Rob Rutten, Henk van der Vorst and Chun Xia for constructive comments on different chapters, Victor Land and Jan Willem Blokland for their contributions to the exercises, Paul Cally, Antoine Cerfon, Bassem Girgis, Pieter Groen, Ian Hutchinson, Ralph Kube and Ryan White for helpful criticism on the previous volumes, and Bram Achterberg, Hubert Baty, Sander Beliën, Nicolas Bessolaz, Tom Bogdan, Fabien Casse, Antoine Cerfon, Paul Charbonneau, Arpád Csík, Herman Deconinck, Peter Delmont, Hans De Sterck, Dan D’Ippolito, Jeff Freidberg, Ricardo Galvão, Marcel Goossens, Giel Halberstadt, Tony Hearn, Bart van der Holst, Hanno Holties, Alan Hood, Wolfgang Kerner, Rob Kleibergen, Max Kuperus, Boon Chye Low, Keith MacGregor, Daniel Mueller, Valery Nakariakov, Ronald Nijboer, Eric Priest, Jan Rem, Bernard Roberts, Andro Rogava, Ilia Roussev, Paulo Sakanaka, Brigitte Schmieder, Karel Schrijver and Lydia Van Driel-Gesztelyi for fruitful collaborations and discussions.

The first author is indebted to Jeff Freidberg for scientific stimulation and friendship for many years. He is also thankful to past and present management of DIFFER (formerly FOM-Institute for Plasma Physcis 'Rijnhuizen'), Marnix van der Wiel, Aart Kleyn, Niek Lopes Cardozo, Noud Oomens, Jan Kranenbarg, Richard van de Sanden, Tony Donné and Marco de Baar, for having provided optimum conditions to work on these books. We wish to thank Simon Capelin of Cambridge University Press for his support and patience over all the years of preparation of this book and the previous volumes. We are indebted to the content manager Roisin Munnelly for helpful assistance and to the copy editor Jon Billam for very careful editing of this book. 